\title{
Mesoscale Upwelling and Density Finestructure in the Seasonal Thermocline-A Dynamical Model
}

\author{
REINER ONKEN \\ Institut für Meereskunde, Kiel, Federal Republic of Germany
}

(Manuscript received 22 January 1991, in final form 24 January 1992)

\begin{abstract}
Results of a three-dimensional primitive equation model are presented simulating turbulent mesoscale motions in the seasonal thermocline on an $f$ plane. The model is based on a hybrid vertical coordinate scheme and conserves isopycnic potential vorticity.

Mesoscale turbulence is modeled in terms of an unstable potential vorticity front. The model integration starts from a purely zonal, $60-\mathrm{km}$-wide geostrophically balanced jet, on which is superimposed a small initial perturbation. The most unstable mode exhibits a wavelength of $85 \mathrm{~km}$ and is driven by a mixed type of instability. Characteristic dynamical ingredients of the wave are enhanced cyclonic and anticyclonic relative vorticity in the troughs and the ridges, respectively, due to the curvature of the flow. Vertical motion of up to $10 \mathrm{~m} \mathrm{~d}^{-1}$ occurring downstream of the ridges (downwelling) and downstream of the troughs (upwelling) is driven by geostrophic advection of relative vorticity. The contrast of static stability across the front is changing during amplification of the instability: in troughs the stability is decreasing whereas in ridges it is increasing. The density field exhibits local anomalies of the isopycnals' depths (bumps) due to the ageostrophic cross-jet advection of potential vorticity streamers wound up in cyclones and anticyclones. Locally, the potential vorticity gradients are enhanced, creating a multiple front structure.

The model results support observations and findings of earlier atmospheric and oceanic models. It is emphasized that mesoscale turbulent structures may have a profound influence on primary productivity, mixed-layer, and internal wave dynamics.
\end{abstract}

\section{Introduction}

Bleck et al. (1988, hereafter referred to as BOW) presented a two-dimensional model simulating the generation of mesoscale potential vorticity fronts in the deformation field of synoptic-scale eddies. They emphasized the role of these fronts in the context of quasigeostrophic turbulence theory (Charney 1971; Rhines 1979) and potential enstrophy cascading to higher wavenumbers of turbulent kinetic energy in the ocean. This theory states that the potential enstrophy cascade is directed from the synoptic scale (typical length scales on the order of $100 \mathrm{~km}$ ) toward the microscale (length scales on the order of $0.1 \mathrm{~m}$ ) where potential enstrophy is annihilated by three-dimensional overturning motions (Salmon 1980). According to Woods (1980) this cascade is nearly divergence-free; thus, the mesoscale waveband embedded between the synoptic scale and the microscale is characterized by turbulent motions transferring the potential vorticity variance (i.e., the potential enstrophy) toward higher wavenumbers. In principle, this process can be achieved

Corresponding author address: Dr. Reiner Onken, Institut fur Meereskunde, Dusternbrooker Weg 20, W-2300 Kiel 1, Federal Republic of Germany. by ever sharpening potential vorticity fronts, but BOW revealed that in purely two-dimensional frontogenesis the nonlinearity of the dynamics prohibits the potential enstrophy from penetrating too far into the mesoscale waveband. This process comes to a halt as soon as the "catchment area" of available potential energy (which is the model domain ) approaches the width of the front defined by the spatial scale of the cross-front ageostrophic circulation. The catchment-area limitation is not simply an artifact of the BOW model design; it also occurs in nature owing to the limited extent of the synoptic-scale eddy deformation fields. Thus, BOW concluded that the continuation of the potential enstrophy cascade into the mesoscale waveband can be achieved only by taking into account three-dimensional effects of frontal dynamics, that is, to permit the front to become unstable. This paper is an extension of the BOW model to include motion in the third dimension. A three-dimensional primitive equation model is presented, which simulates the next step in the enstrophy cascade after frontogenesis-the development of waves on a potential vorticity front.

Why is there a need for models of mesoscale dynamics? First of all, these models give insight into a spectral band of oceanic motion that at present is parameterized only in gyre-scale circulation models (GSCM) by a rather arbitrarily chosen eddy coefficient. Recent mod- 
els (for example, Cox 1987) are only able to resolve the low-wavenumber end of those length scales that are important for the generation of synoptic-scale eddies: the internal Rossby deformation radius. Today's models have at best a $1 / 6^{\circ}$ horizontal resolution. This gives us some confidence that the dynamics and the role of eddies larger than about $100 \mathrm{~km}$ may be modeled rather well. But the spectrum of turbulent motion in the ocean extends farther to higher wavenumbers. We know about the existence of smaller eddies (e.g., McWilliams 1985), which emphasizes the need to resolve down to $O(1) \mathrm{km}$ in order to model accurately the influence of the eddy motion on the gyre-scale circulation and, for example, the meridional heat transport. For GSCMs this requires the resolution to be increased by one order of magnitude. This means the number of grid points has to be multiplied by two and the run time by three orders of magnitude. Computers powerful enough to manage such models will probably not be available within the next decade. Hence, if we want to learn more about the role of this subgrid-scale turbulent motion we have to concentrate on high-resolution mesoscale models. These models offer the opportunity to improve our knowledge about many mesoscale features, some of which will be addressed in this study.

Mesoscale upwelling is a phenomenon of first-order importance for primary production in the ocean. Vertical motions raise nutrients into the euphotic zone, enabling the growth of phytoplankton. Thus, we may guess plankton patchiness to be a consequence of local upwelling events related in a systematic way to mesoscale turbulent structures. Several model studies exist that provide information about the strength and the locations of up- and downwelling in unstable waves (Saltzman and Tang 1975; Kielmann and Käse 1987; Oey 1988), but no explanation is offered concerning the mechanism driving the vertical motion. Another mesoscale feature of interest is the density finestructure in the vicinity of fronts. Although we have some ideas what the density field is like across a front, these ideas are mainly based on two-dimensional models like BOW, but as soon as fronts become unstable we have to take into account the three-dimensionality of the motion field and cannot expect density finestructure to be explained satisfactorily by two-dimensional arguments. This has already been demonstrated by Joyce (1977) and Woods et al. (1986), who explained thermohaline interleaving in frontal regions simply by the advection of temperature along isopycnic surfaces rather than making any farfetched assumptions in order to explain it as the result of double-diffusive processes.

In this paper a three-dimensional model is presented to examine mesoscale dynamics. Assuming typical length scales of $10 \mathrm{~km}$ and velocities of $0.1 \mathrm{~m} \mathrm{~s}^{-1}$, mesoscale dynamics is characterized by a Rossby number of order 0.1 . Thus, primitive equations are used in the model. Mesoscale structures exhibit strong horizontal variability (cf. Flament et al. 1985); this is taken into account by using high horizontal resolution. Conservation of isopycnic potential vorticity, the key constraint of the potential enstrophy cascade, is taken into account by adopting the Bleck and Boudra (1981, hereafter referred to as BB) model code based upon a hybrid vertical-coordinate scheme.

A description of the model and the initial conditions is given in section 2 . The model results are presented in section 3, which describes first the spectral response of the model to a white-noise initial perturbation and then how the most unstable wave has been found. In a second model run the properties of this wave will be investigated: energetics, growth rates and phase speeds, patterns of upwelling, relative and potential vorticity, and structures of the density field and layer-thickness modulation. An explanation of the observed features by using potential vorticity conservation arguments will be given in section 4 . Section 5 contains the discussion and a comparison of the model results with other models and observations. Finally, a summary and the conclusions can be found in section 6 .

\section{The model}

As already pointed out in the Introduction, this model is based upon the $\mathrm{BB}$ primitive equation model using a hybrid vertical coordinate. "Hybrid" means that the vertical coordinate is isopycnic in most parts of the model domain, but it can become isobaric where coordinate surfaces tend to intersect each other. This may happen, for example, at the sea surface (which is also a coordinate surface), or in upwelling regions. The governing equations, the model code, and the nomenclature are identical to BB. Only deviations from that model will be reported in this section.

\section{a. Boundary conditions, technical details, and parameters}

The model domain is a rectangular box limited by solid walls at $x=0$ and $x=L$ and in $y$ direction at $y$ $=0$ and $y=B$, where $x$ and $y$ are the horizontal Cartesian coordinates; $x$ is positive to the south and $y$ positive to the east, $L$ will be referred to as cross-front scale and $B$ as alongfront scale. The bottom of the box is at $p$ $=1000 \mathrm{dbar}$. The boundary conditions at $x=0$ and $x=L$ are $u=0$ and $\partial v / \partial x=0$ (free slip), $u$ and $v$ being the components of the horizontal velocity vector in $x$ and $y$ direction, respectively. In the $y$ direction periodic boundary conditions are applied. The boundary conditions for the bottom and the sea surface are those for material surfaces; that is, $\dot{s} \partial p / \partial s=0$ at $p=0$ and $p=1000 \mathrm{dbar}$. Here $p$ is pressure and $s$ is the generalized model vertical coordinate. To filter out fastmoving gravity waves a rigid-lid condition is applied. There is no diabatic heating/cooling in the interior of the box. 
In both horizontal directions the model is divided up into 128 equally spaced grid intervals $\Delta x=\Delta y=2$ $\mathrm{km}$. In the vertical it has five layers. The minimum layer thickness allowed to be attained during the model integration has been set to $\Delta p_{0}=3 \mathrm{dbar}$. A biharmonic eddy viscosity formulation [cf. BB (6)] is applied to reduce small "numerical" waves, using a coefficient $\nu$ $=10 \mathrm{~m}^{2} \mathrm{~s}^{-1}$. Together with the horizontal grid size this yields an effective viscosity $\nu^{*}=\nu \Delta x \cdot \Delta y=4 \times 10^{7}$ $\mathrm{m}^{4} \mathrm{~s}^{-1}$. Thus, for waves longer than two grid increments the damping effect is very much less than in the monoharmonic formulation. A $20-\mathrm{km}$ wave, for example, is damped on a time scale of $\sim 100$ days, whereas in the monoharmonic formulation, the damping time scale is $\sim 10$ days using the same value of $\nu$. High-frequency fluctuations of the velocity field are damped by a three-point $(0.25-0.5-0.25)$ time smoother. The Coriolis parameter $f=10^{-4} \mathrm{~s}^{-1}$ is constant ( so far, it is unnecessary to assign the geographic directions south and east to the horizontal coordinates $x$ and $y$, respectively; but these assignments have been retained, because it will facilitate the description of the model results). The time step is $400 \mathrm{~s}$.

\section{b. Initial conditions}

This paper is intended to investigate structures of unstable fronts owing to baroclinic or barotropic instability. Thus, the initial conditions should meet known necessary conditions for instability in simplified systems. A necessary condition for baroclinic instability is a potential vorticity gradient changing sign somewhere with depth. Barotropic instability requires a sign change of the horizontal gradient of the absolute vorticity (Pedlosky 1979). The two-dimensional front created by the BOW model after three days of integration satisfies both conditions: Fig. 8 in BOW demonstrates the barotropic instability condition ( $f$ plane!), but the baroclinic instability condition is only poorly met. This is due to the circumstance that nonzero differences of isopycnic potential vorticity occur only in the top and bottom layer, but in the latter the difference is one order of magnitude less. I have tested the BOW front as initial condition for this model and found it indeed to be only weakly unstable. In these tests the full BOW vertical resolution was used with ten layers before I reduced the number of layers in this study; thus, the stability of the BOW model is not caused by the vertical resolution. The reason for the lower vertical resolution is the fact that in later stages of meander growth the layer thickness due to compression of vortex tubes falls below the critical distance of $\Delta p_{0}$ (see the following). This gives rise to water exchange across $s$ surfaces, and isopycnic potential vorticity is no longer conserved. In order to create an initial density field satisfying better the condition for baroclinic instability, the BOW initial field has been modified slightly as follows.

The selected initial condition is $y$ independent. The pressure variation on the $k$ th surface (which is an isopycnal at $t=0$ ) is defined as

$$
\begin{aligned}
p_{k}(x)=p_{k} & (x=0) \\
& +[\tanh (c x / L-c / 2)+1] \Delta p_{\max _{k}} / 2 ;
\end{aligned}
$$

$k$ runs from 1 to 6 . Surfaces 1 (top) and 6 (bottom) are defined to be horizontal, and $\Delta p_{\max _{k}}$ is the maximum horizontal pressure variation on the $k$ th surface between $x=0$ and $x=L$ (in fact, it is the pressure variation between $x=-\infty$ and $x=+\infty$ ). The pressure values $p_{k}$ at $x=0$ and the maximum horizontal pressure variation for $s$ surfaces 1 to 6 and the average $s$ layer densities $\sigma_{t_{k}}=1 / \alpha_{t_{k}}-1000$ for $s$ layers 1.5 to 5.5 are summarized in Table 1, where $\alpha$ means specific volume. (The numbering system for coordinate layers has been chosen to indicate the relation between layers

TABLE 1. Initial density field parameters of the model (bold) and the corresponding values of the BOW model (light).

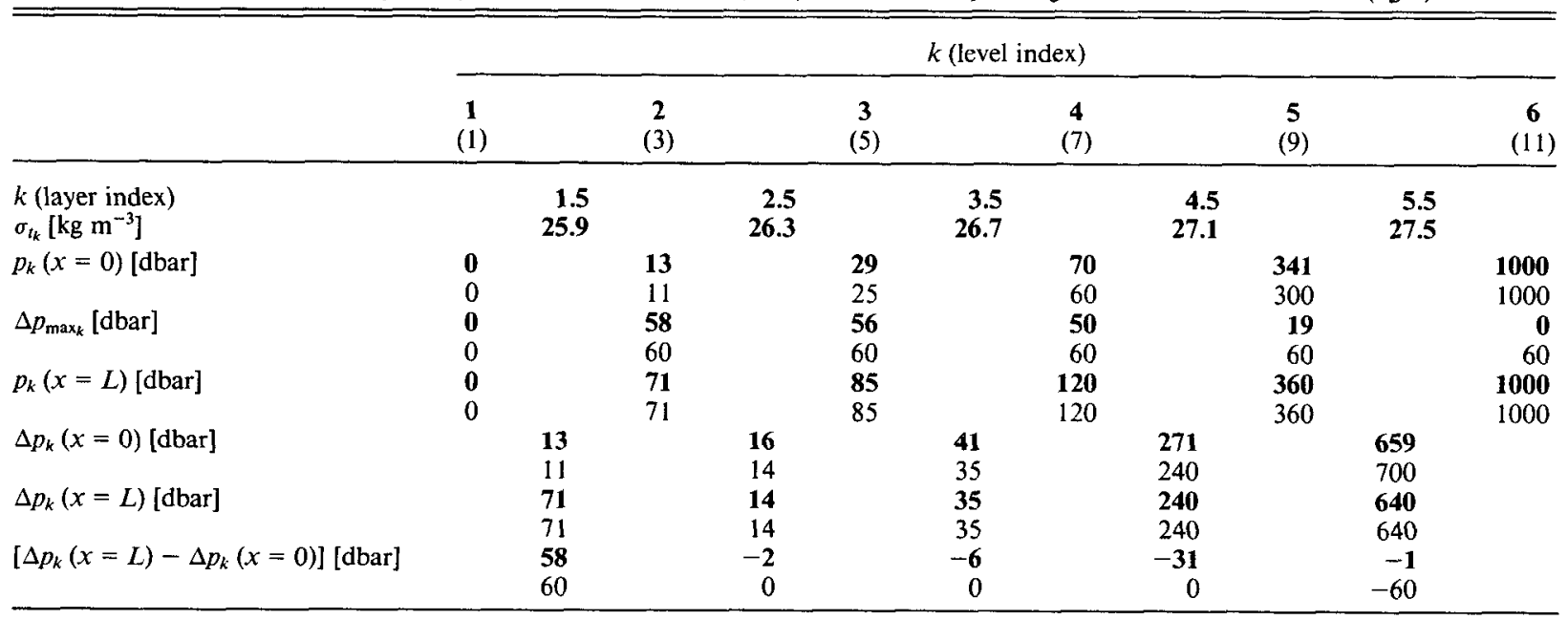


and levels.) The parameter $c$, controlling the sharpness of the hyperbolic tangent function, has been set to 14 . Equation (1) is identical with the equation in BOW, section 5, defining the initial pressure field there (note that the origin in BOW is shifted by half the channel width). In order to compare the initial conditions of BOW and the present model, $p_{k}(x=L)$, which is the level pressure at $x=L$, and the corresponding layer thickness values $\Delta p_{k}$ at $x=0$ and $x=L$ are included in Table 1 . On every $s$ surface the pressure at $x=L$ is
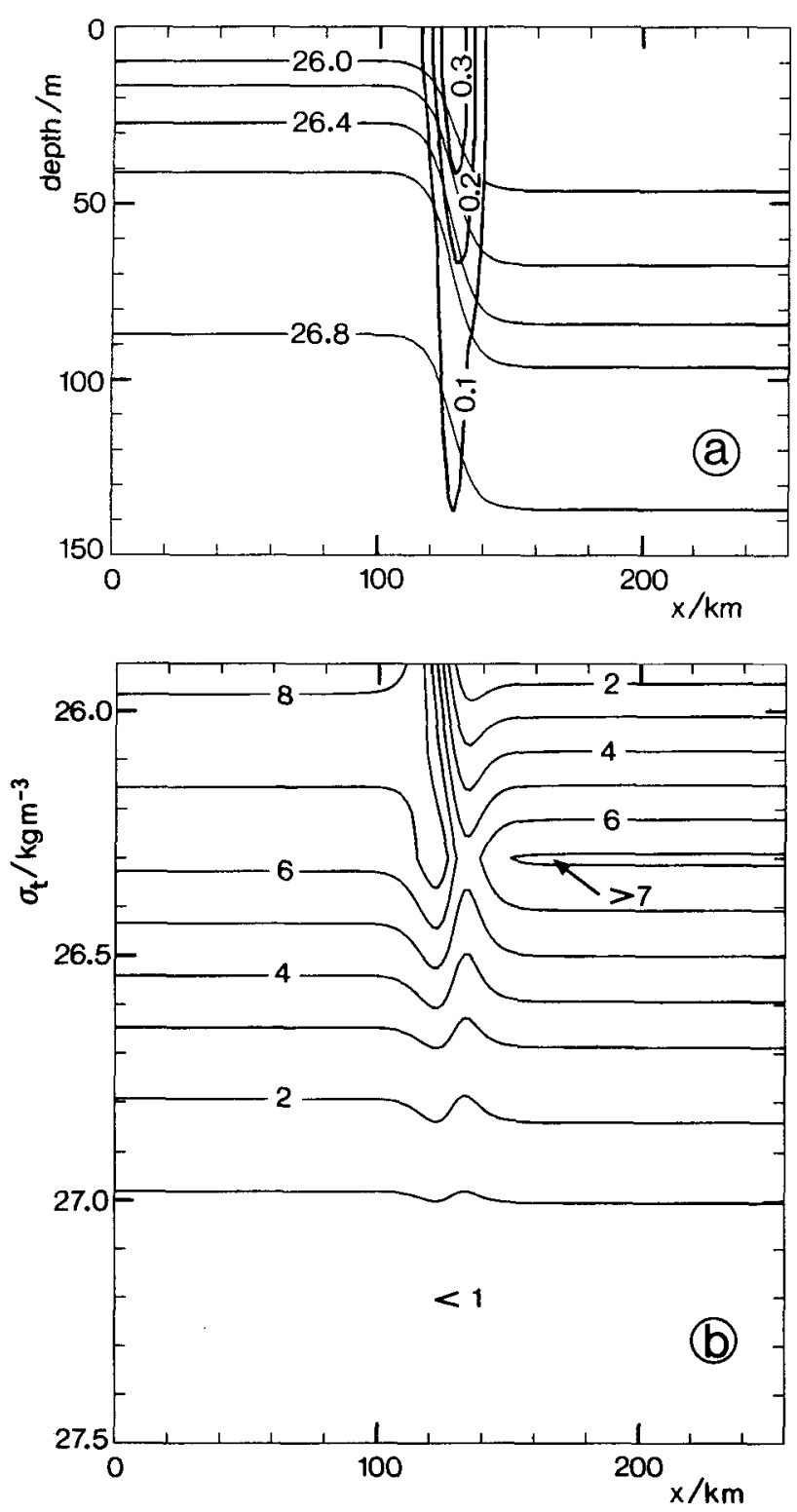

FIG. 1. Cross sections of initial fields. (a) Density and geostrophic velocity field in the upper 150-m depth range. Isopycnals (in $\sigma_{t}$ units) are indicated by thin lines, jet isotachs (in $\mathrm{m} \mathrm{s}^{-1}$ ) by thick lines. Contour interval is $0.2 \mathrm{~kg} \mathrm{~m}^{-3}$ and $0.1 \mathrm{~m} \mathrm{~s}^{-1}$, respectively. (b) Isopycnic potential vorticity (in units of $10^{-6} \mathrm{dbar}^{-1} \mathrm{~s}^{-1}$ ). Contour interval is 2.5 . the same in BOW and in the present model. But the slope, that is, the maximum cross-channel pressure change, decreases with depth in the present model, whereas in the BOW model it has a constant value. This leads to decreasing layer thickness between $x$ $=0$ and $x=L$ in layers $2.5-5.5$, or an equivalent increase of isopycnic potential vorticity. Together with the opposite sign of the potential vorticity gradient in layer 1.5, this initial field satisfies the necessary condition for baroclinic instability in a stronger way than BOW did.

Figure la shows a cross section of the initial density field and the geostrophic alongchannel velocity. Horizontal and vertical scales of the jet defined by the extent of the $0.1 \mathrm{~m} \mathrm{~s}^{-1}$ isotach are about $25 \mathrm{~km}$ and $140 \mathrm{~m}$, the maximum speed at the sea surface is close to 0.4 $\mathrm{m} \mathrm{s}^{-1}$, and the internal Rossby radius of deformation is $11 \mathrm{~km}$. A cross section of the initial potential vorticity field using density as vertical coordinate is shown in Fig. $1 \mathrm{~b}$. The units $\mathrm{s}^{-1} \mathrm{dbar}^{-1}$ used in the figure caption arise from the fact that potential vorticity in the model is represented by the expression $(\partial v / \partial x-\partial u / \partial y+f) /$ $(\partial p / \partial s)$.

\section{Model results \\ a. Finding the most unstable wavelength}

The initial conditions represent the basic state in the sense of instability theory. Meanders on this front can form and grow only if a perturbation is superimposed, because the density field is exactly identical in every cross-front section. Here the properties of the most unstable wave will be investigated, which has been found in the following way: An ageostrophic barotropic whitenoise random perturbation containing all possible wavenumbers has been defined by means of an initial $u$ field (zero so far) varying sinusoidally in the $y$ direction:

$$
\begin{aligned}
u=u_{0}\left[a _ { 1 } \operatorname { e x p } \left(a_{2} \frac{x}{L}\right.\right. & \left.\left.+a_{3} \frac{x^{2}}{L^{2}}\right)-a_{1}\right] \\
& \times \sum_{i=1}^{64} \sin \left(2 \pi \cdot \kappa_{i} \cdot \frac{y}{B}+\varphi_{i}\right) .
\end{aligned}
$$

Here, $u_{0}=1 \mathrm{~mm} \mathrm{~s}^{-1}$ is the single Fourier component initial perturbation amplitude, $\kappa_{i}=B / \lambda_{i}$ the channel wavenumber, $\lambda_{i}$ the corresponding wavelength, and $\varphi_{i}$ the random phase in the range $0<\varphi_{i}<2 \pi$. The function enclosed in brackets using the coefficients $a_{1}$ $=10^{-3}, a_{2}=4 \ln a_{1}$, and $a_{3}=-a_{2}$ is symmetric about $x / L=0.5$. There it has a maximum value of nearly 1 and decays monotonically toward zero when approaching the side walls. Hence, the perturbation is strongest where the jet has its maximum speed, and does not violate the solid-wall boundary condition at $x=0, L$. 


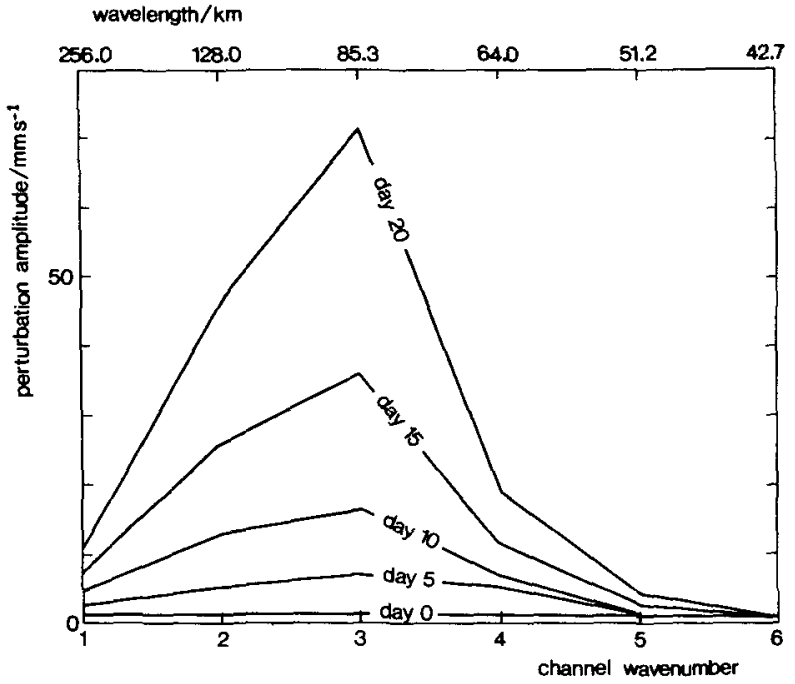

FIG. 2. Wavenumber spectra of the perturbation amplitude in successive 5 -day intervals.

I integrated the model over 20 days and performed a Fourier analysis of the cross-channel velocity $u$ in layer 1.5 at $x=128 \mathrm{~km}$ in successive 5-day intervals. The corresponding amplitude in the wavenumber range $1 \leqslant \kappa_{i} \leqslant 6$ is shown in Fig. 2 . No growth occurs for wavenumber 6 and beyond; thus, the cutoff wavelength is somewhere between 51.2 and $42.7 \mathrm{~km}$. The fastest-growing mode is wavenumber 3 , representing a wavelength of $85.3 \mathrm{~km}$. The amplitude of wavenumber 3 increases from $1 \mathrm{~mm} \mathrm{~s}^{-1}$ on day 0 to over $70 \mathrm{~mm} \mathrm{~s}^{-1}$ on day 20. This finding is consistent with the linear two-layer calculations presented by Killworth et al. (1984, hereafter referred to as KPS). For an internal Rossby radius of $11 \mathrm{~km}$, KPS predict for the $r=5$ case (total water depth about five times the thickness of the upper layer) the most unstable mode to have a wavelength of $84 \mathrm{~km}$ and a cutoff wavelength of $61 \mathrm{~km}$.

\section{b. Properties of the most unstable wave}

\section{1) HORIZONTAL FLOW FIELD}

In order to investigate the physical properties of the most unstable wave, a second model run of over 35 days has been performed containing only wavenumber 3 in the perturbation field given by (2). The final streamfunction at the sea surface (layer 1.5 ) is displayed in Fig. 3a. In this figure (and also in Figs. 6, 8, 9a, 10a, and 12) only the range between $72 \mathrm{~km}<x<184 \mathrm{~km}$ is shown, because nothing interesting happens in the outer regions of the model domain. The streamfunction has been derived by integrating the relative vorticity twice; thus, only the divergence-free part of the total flow field is displayed here. As we shall see later, the deviation of the total flow from the streamfunction does not exceed $5 \%$; hence, the streamfunction is a rather well-suited quantity to give a first impression of the horizontal flow field. Within 35 days, meanders have developed and disturbed the initial, strictly zonal orientation of the front. The meander amplitude (i.e., the amplitude of the streamlines) has grown to about $20 \mathrm{~km}$ within this time. The streamfunction gradient reveals inequalities in the flow around troughs and ridges. The stronger gradient in the troughs indicates enhanced flow here, whereas the separation of the streamlines in the ridges demonstrates that here the flow becomes weaker. This is confirmed by Figs. 3b,c showing sections of the along-channel velocity component in a trough and a ridge. In the trough (Fig. 3b) the width of the jet defined by the extent of the 0.05 $\mathrm{m} \mathrm{s}^{-1}$ isotach is about $60 \mathrm{~km}$. The maximum speed at the sea surface is $0.398 \mathrm{~m} \mathrm{~s}^{-1}$, that is, nearly the same as the initial value. In the ridge, however, the jet width is $100 \mathrm{~km}$, and the maximum speed has dropped to $0.294 \mathrm{~m} \mathrm{~s}^{-1}$. In addition, the jet has become asymmetric. The horizontal extent of the anticyclonically sheared side is nearly twice as large as the extent of the cyclonically sheared side.

\section{2)ENERGETICS}

First, the question will be answered as to which type of instability has led to the observed meander growth on the front by means of evaluating the conversion rates between mean and eddy components of potential and kinetic energy. I used the same formulation of energy conversions as Boudra et al. (1988) in their isopycnic model of instabilities in the Florida Current. Though my model is in principle nonisopycnic, this formulation can be applied because I have verified that all $s$ surfaces coincide with isopycnals at all times of the model integration. Figure 4 displays time series of energy conversions during the 35-day model run. Here $\mathrm{PK}=\mathrm{PK}+\mathrm{PK}^{\prime}$ represents the conversion from potential to kinetic energy, where $P \bar{K}$ is the conversion from potential to (temporal) mean kinetic and PK' the conversion from potential to eddy kinetic energy. According to Boudra et al. (1988) and Bleck and Boudra (1986), $\mathrm{PK}^{\prime}$ is the quantity representing baroclinic instability in isopycnic coordinates. During the first 20 days or so of the model integration, all three quantities are close to zero. Later PK begins to rise continuously, until it reaches a final value of about $0.27 \mathrm{~mW} \mathrm{~m}^{-2}$ on day 35 . This rise can be decomposed into a strong increase of $\mathrm{PK}^{\prime}$ and a less strong decrease of $\mathrm{PK}$, which means that while the reservoir of potential energy feeds the eddy kinetic energy, a fraction of this potential energy loss is balanced by conversion of mean kinetic energy. In addition to this "indirect" route of converting kinetic energy of the mean flow to eddy kinetic energy via the potential energy, there is also the direct route $\overline{\mathrm{K}} \mathrm{K}^{\prime}$, representing the conversion of mean kinetic to eddy potential energy by the action of Reynolds stresses. This quantity is always positive except after 

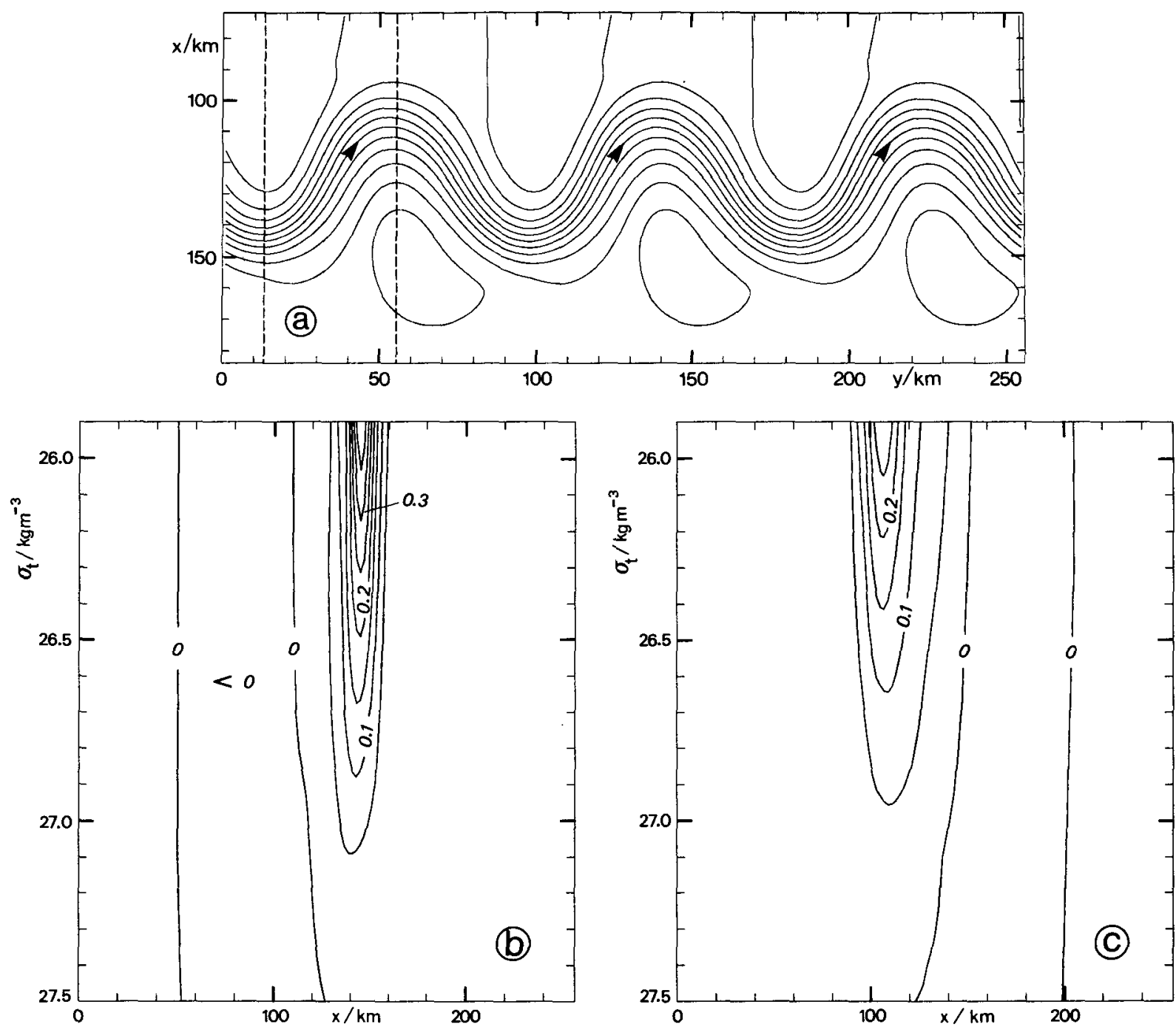

FIG. 3. The horizontal flow field in layer 1.5 on day 35. (a) The streamfunction. Arrows indicate the direction of the flow. Contour interval is $500 \mathrm{~m}^{2} \mathrm{~s}^{-1}$. Broken lines indicate positions of cross sections. (b), (c) Cross sections of the along-channel velocity at (b) $y=13$ $\mathrm{km}$ and (c) $y=55 \mathrm{~km}$. Contour interval is $0.05 \mathrm{~m} \mathrm{~s}^{-1}$. The positions of the sections are indicated in Figs. 3a, 8, 9a, and 10a.

day 34 , which means that the eddy kinetic energy reservoir is also fed by the barotropic instability mechanism. During the first time period of significant energy conversion, say between days 20 and about 25 , PK' and $\overline{\mathrm{K}} \mathrm{K}^{\prime}$ are the same order of magnitude; thus, both baroclinic and barotropic instability are contributing to the meander growth. This mixed type of instability is what we would expect from the theoretical results of Killworth (1980), because the width of the jet is about the same order of magnitude as the Rossby radius and the stratification is confined to a small fraction of the total depth. Later on, $\overline{\mathrm{K}} \mathrm{K}^{\prime}$ decreases and becomes even negative after day 34 , indicating that now the eddies begin to stabilize the mean flow. As PK' continues to increase during that period of time, the instability becomes a pure baroclinic one.

\section{3) GROWTH RATES AND PHASE SPEEDS}

In the present model run I also performed a Fourier analysis of the $u$ field at $x=128 \mathrm{~km}$ in 1-day intervals and evaluated amplitudes, growth rates, and phase speeds of the unstable wave propagating through the channel. The temporal evolution of the $u$ amplitude and the corresponding growth rate in layer 1.5 is displayed in Fig. 5a. The amplitude grows from $1 \mathrm{~mm} \mathrm{~s}^{-1}$ at the beginning to about $250 \mathrm{~mm} \mathrm{~s}^{-1}$ after 35 days. Initially, there is strong exponential growth starting at a rate of about $0.63 \mathrm{~d}^{-1}$. But the rate decreases rapidly and stays at a nearly constant level of about $0.16 \mathrm{~d}^{-1}$ between about day 10 and day 20 . Later on the growth rate decreases again and reaches a final value of about $0.02 \mathrm{~d}^{-1}$ at the end of the model run. A qualitatively 


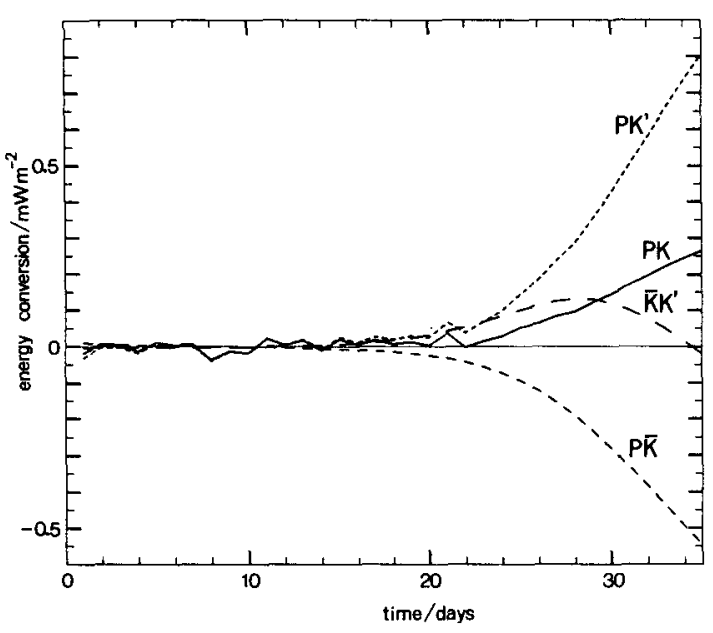

FIG. 4. Time series of energy conversions integrated over the whole model domain. PK: Conversion of potential to kinetic energy, PK': conversion of potential to eddy kinetic energy, $\mathrm{PK}$ : conversion of potential to temporal-mean kinetic energy, $\overline{\mathrm{K}} \mathrm{K}^{\prime}$ : conversion of temporal-mean kinetic to eddy kinetic energy.

similar growth-rate behavior could also be found in Wood's (1988) nonlinear model. The growth rate has also been compared with KPS's results, but they predict for the $r=5$ case a linear growth rate of only $0.4 \mathrm{~d}^{-1}$. I have no explanation for this discrepancy.

The temporal evolution of the phase speed in layers $1.5,3.5$, and 5.5 is displayed in Fig. $5 b$. At the beginning, the phase of the wave is the same in all layers due to the initialization by (2). But as soon as the integration starts, the phases become different in each layer and begin to adjust to a constant vertical phase lag. This adjustment process is finished after about 4 days in layers 1.5 and 3.5 , but it requires approximately 16 days in the bottom layer. After adjustment the wave in every layer travels at a constant speed of $5 \mathrm{~cm} \mathrm{~s}^{-1}$ through the channel. At that instant, the phase lag between top and bottom is about $\pi / 5$. This phase difference is a typical feature for baroclinically unstable waves since this enables the extraction of potential energy to be converted to perturbation kinetic energy. After about day 25 the phase speed decreases-an indication for the beginning influence of nonlinear effects. For the phase speed a comparison with KPS gives better agreement than for the growth rate; KPS predict a phase speed of $6.16 \mathrm{~cm} \mathrm{~s}^{-1}$, about $20 \%$ higher than my value. However, there is a qualitative difference between the temporal evolution of the phase speed in comparison with Wood's (1988) model, where the phase speed decreases rapidly with time in the same way as the growth rate does (cf. his Fig. 5). I believe this discrepancy is due to the method of evaluating the phase speed. Wood's calculations were based on the speed of the trough and crest of the wave (method I). Here, the phase speed is calculated halfway between the trough and crest in the center of the channel; that is, it is based on the intersection point of the streamlines with the $y$ axis (method II). Ikeda (1981) tested both methods in his model and found that method I yields a rapid decrease of phase speed, whereas the speed was nearly constant using method II.

\section{4) VERTICAL MOTION}

The vertical motion $w$ is a quantity diagnosed on $s$ surfaces. Figure 6 shows $w$ at level 2 on day 35. Upand downwelling areas are clearly separated. Upwelling occurs in front of the troughs, downwelling behind
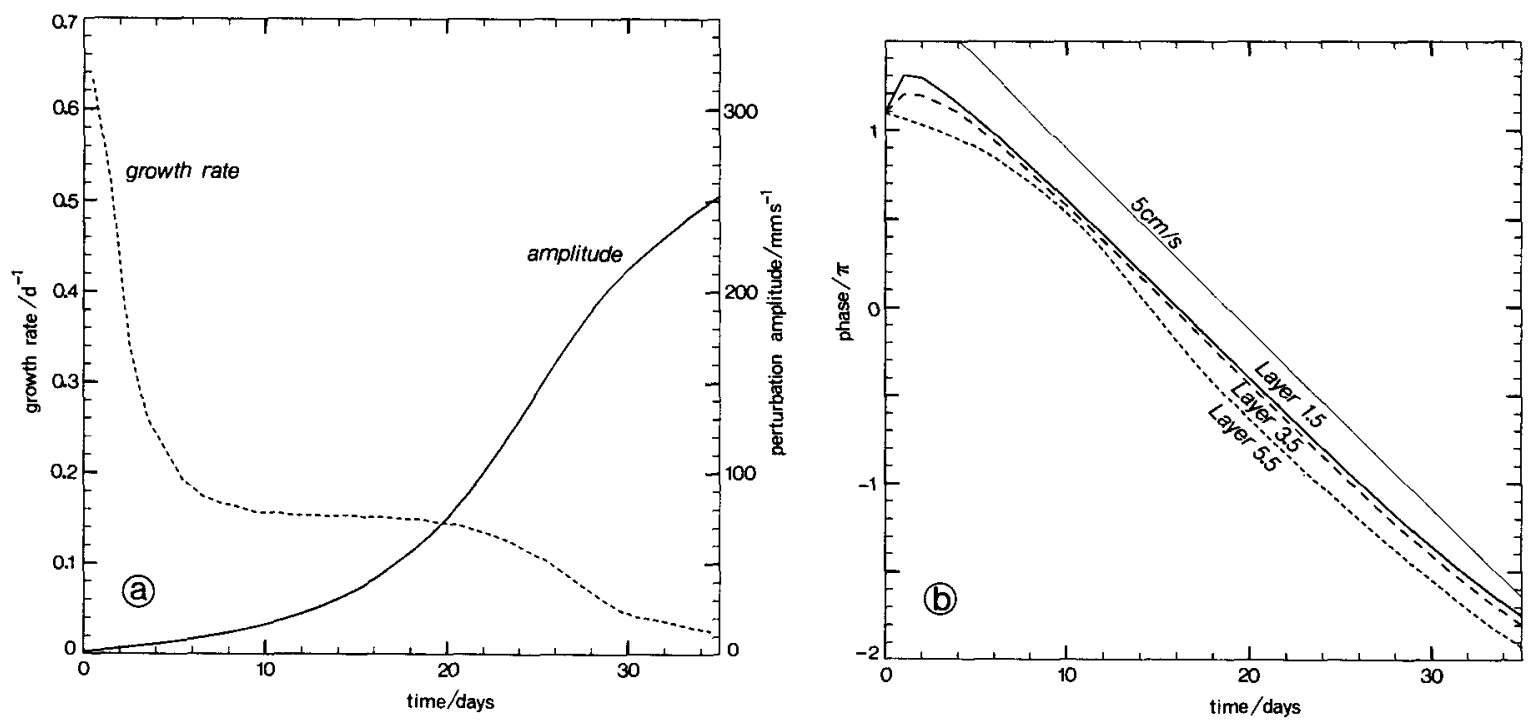

FIG. 5. (a) The evolution of the perturbation amplitude and the growth rate of channel wavenumber 3 . (b) The phase (normalized by $\pi$ ) of channel wavenumber 3 as a function of time in model layers 1.5, 3.5, and 5.5. For comparison, the thin straight line representing a phase speed of $5 \mathrm{~cm} \mathrm{~s}^{-1}$ has been added. 


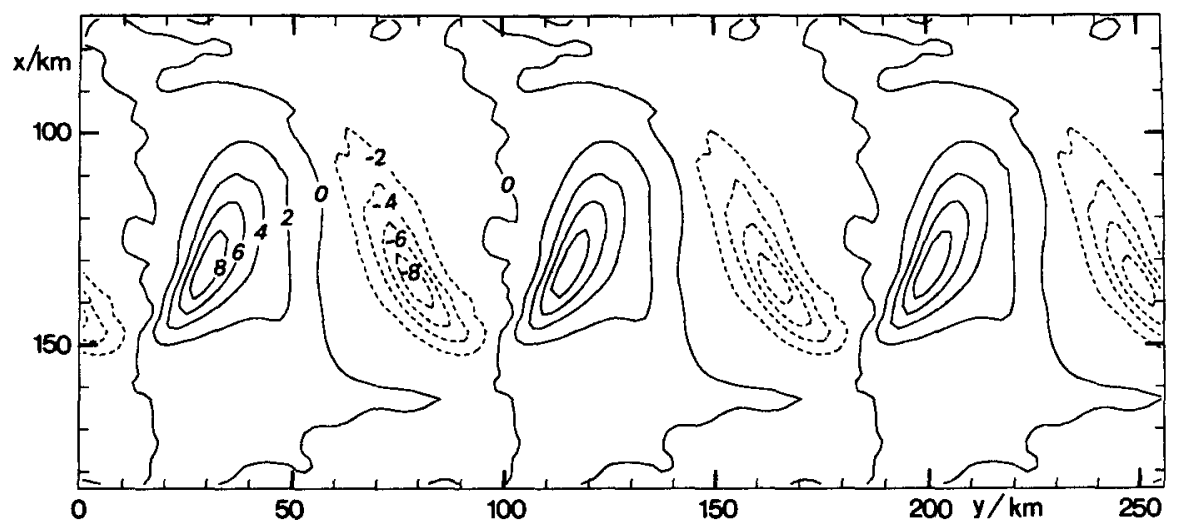

FiG. 6. The field of vertical motion $\left(\mathrm{m} \mathrm{d}^{-1}\right.$ ) at level 2 (mean depth $41.5 \mathrm{~m}$ ) on day 35 . Solid contours refer to upwelling, broken contours to downwelling. The zero line is the first solid one. The contour interval is $2 \mathrm{~m} \mathrm{~d}^{-1}$.

them (cf. Fig. 3). Maximum upwelling speeds are 9.3 $\mathrm{m} \mathrm{d}^{-1}$; extreme downwelling speeds are $-9.6 \mathrm{~m} \mathrm{~d}^{-1}$. The intensity of $w$ increases continuously during the entire model run (not shown).

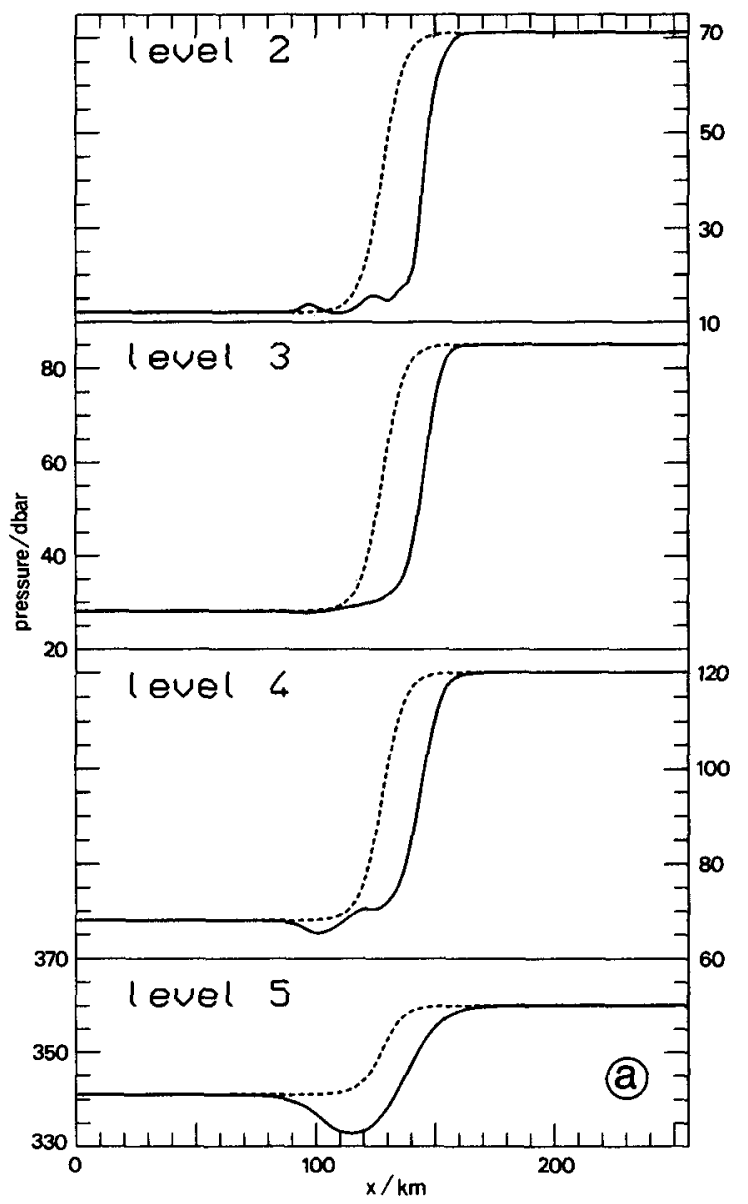

\section{5) CROSS-FRONT PRESSURE DISTRIBUTION}

Figure 7 shows the cross-channel pressure distribution on density surfaces (which are equivalent to model

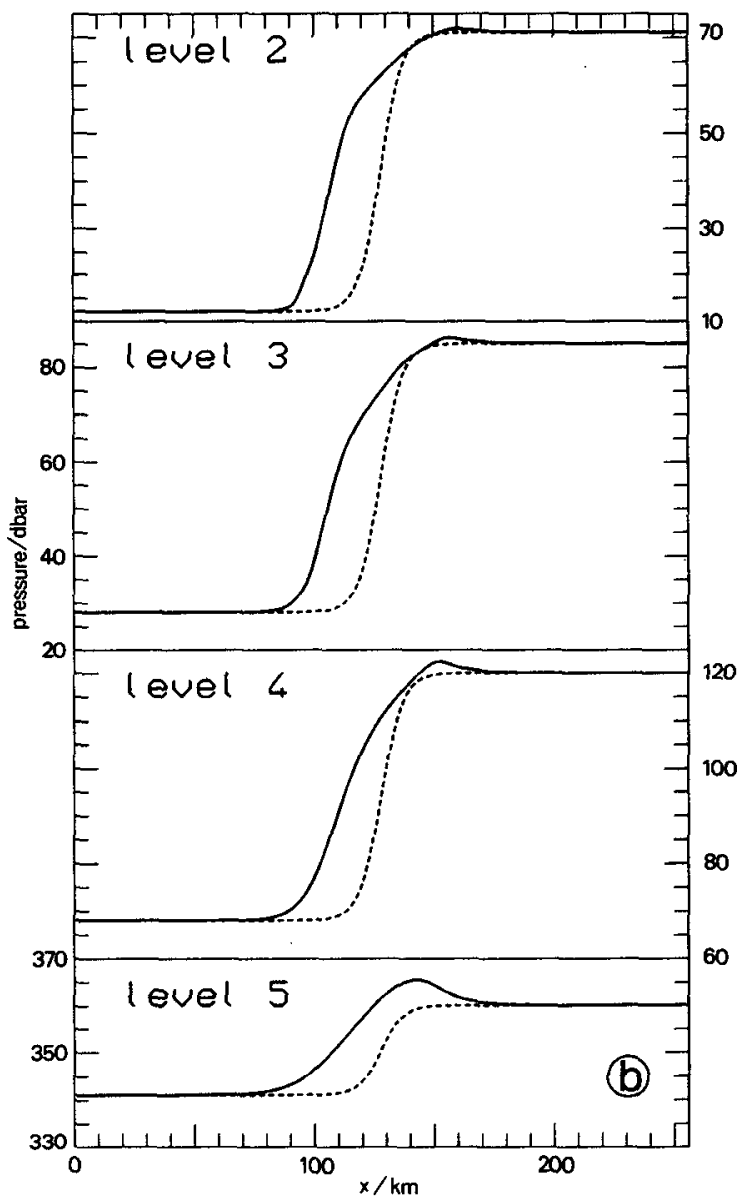

FIG. 7. Cross sections of pressure on $s$ levels on day 35 (solid lines) and day 0 (broken lines): (a) at $y=13 \mathrm{~km}$, (b) at $y=55 \mathrm{~km}$. The positions of the sections are indicated in Figs. 3a, 8, 9a, and 10a. 
levels) on day 35 and for comparison at the beginning of the integration. The pressure distribution in a trough (Fig. 7a) reveals that the front has moved to the right by about $20 \mathrm{~km}$. The cross-front pressure gradient has not changed significantly. This is also reflected by the unchanged maximum jet speed assuming nearly geostrophic balance. In the ridge (Fig. $7 \mathrm{~b}$ ) the front has moved about $20 \mathrm{~km}$ to the left. In contrast to the trough, however, the pressure gradient has become weaker with progressing time, especially on the righthand side of the front, giving it an asymmetric shape. This is also in agreement with the weaker jet speed in the ridges and the asymmetry of the jet shear. In addition to these temporal changes of the large-scale pressure distribution, we also observe some finestructure features, which have grown during the model integration. In Fig. 7a at levels 2 and 4 on the left-hand side of the main front, the initial hyperbolic tangent shape of the isopycnals has been distorted by little "bumps." These bumps have a vertical scale of only a few meters; the horizontal wavelength is about $20 \mathrm{~km}$. Features with similar horizontal and vertical scales can also be seen in Fig. 7b at levels 2, 3, and 4, but on the right-hand side of the main front. Another type of pressure anomaly is encountered at level 5. Both in the trough on the left-hand side of the front and in the ridge on the right-hand side the isopycnals have been displaced toward the bottom (trough) or the sea surface (ridge) by about 7 dbar. But the horizontal scales of these anomalies are about five times larger than the scales of the bumps. This leads to the conjecture that they have been created by different physical mechanisms.

\section{6) RELATIVE VORTICITY}

The direction of the initial jet was strictly parallel to the channel walls, thus the relative vorticity $\zeta=(\partial v /$ $\partial x)_{s}-(\partial u / \partial y)_{s}$ on day 0 also was only dependent on $x$. Extreme values of $\zeta / f$ were \pm 0.31 . Figure 8 shows contours of $\zeta / f$ in layer 1.5 on day 35 . The initially parallel bands of cyclonic and anticyclonic relative vorticity are now separated into closed "banana"shaped cells of either sign. Extreme values are 0.66 located in the peaks of the troughs and -0.42 on the anticyclonically sheared side of the jet, where the streamline curvature changes sign.

\section{7) LAYER THICKNESS ANOMALY}

Potential vorticity conservation requires a balance between changes of relative vorticity and the vertical separation between isopycnals (i.e., the layer thickness). Figure 9a shows contours of $H^{\prime}=H-\vec{H}$ on day 35 in layer 2.5 , where $H$ is the actual layer thickness and $\bar{H}$ the thickness of this layer averaged over the whole model domain. For continuity reasons $\bar{H}$ is constant in time as long as no transport of fluid has taken place across $s$ surfaces. This has never happened during the integration of the model. Extreme values of $H^{\prime}$ on day 0 have been $-1 \mathrm{dbar}$ and $1 \mathrm{dbar}$. On day 35 these extrema are -3.4 and $6.8 \mathrm{dbar}$. Positive anomalies are confined to the troughs, whereas negative contours can be found in regions of anticyclonically rotating flow. At the northern edge of the cyclones a thin ribbon of negative thickness modulation $H^{\prime}<-1$ dbar can be seen.

In order to demonstrate local changes of layer thickness I have added cross sections of layer thickness through a cyclone (Fig. 9b) and an anticyclone (Fig. $9 \mathrm{c})$. In both figures, levels 1.5 and 5.5 have been omitted, because information on the thickness of these layers can be drawn already from the pressure distribution on levels 2 and 5, respectively, shown in Figs. 7a,b. In Fig. $9 \mathrm{~b}$ the most dramatic changes of layer thickness can be encountered at about $x=140 \mathrm{~km}$. In layer 2.5 , for example, the thickness has increased to about 22 dbar, though initially the overall maximum thickness

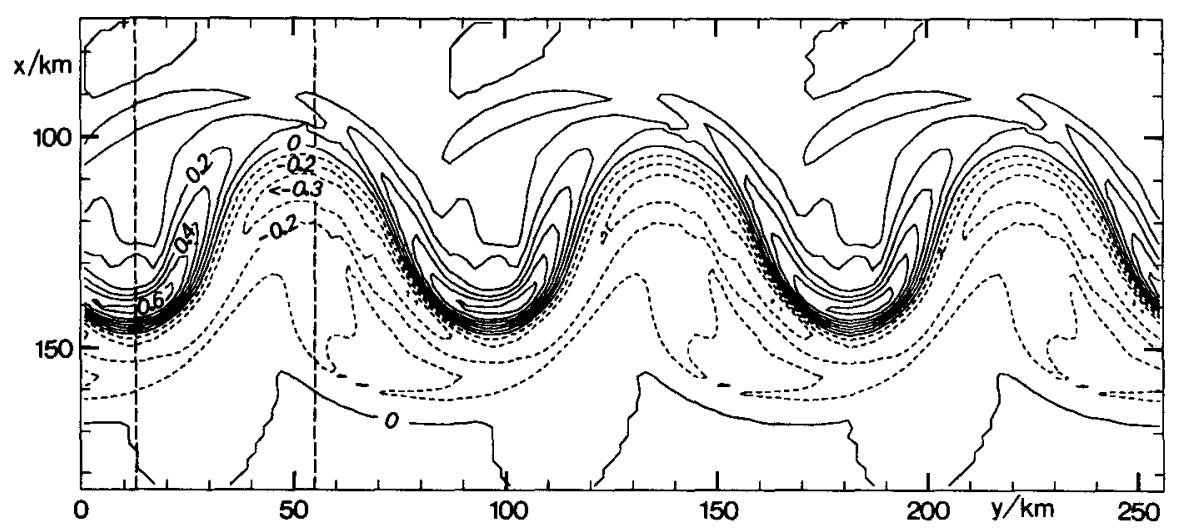

FIG. 8. The field of relative vorticity normalized by the planetary vorticity on day 35 in layer 1.5. Solid contours refer to cyclonic, broken contours to anticyclonic relative vorticity. The zero line is the first solid one. Contour interval is 0.1 . Broken lines indicate positions of cross sections. 

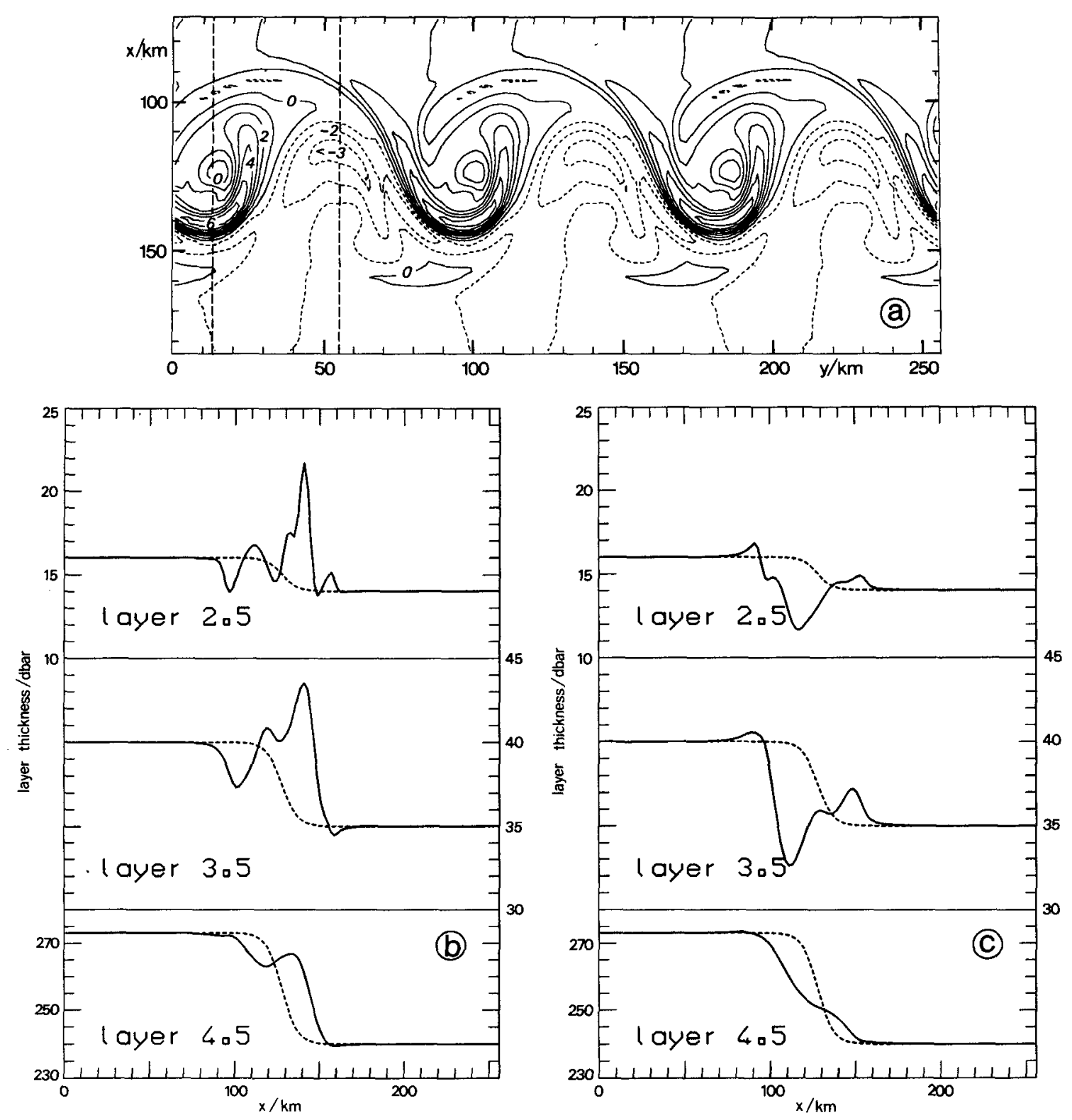

FIG. 9. (a) The layer-thickness anomaly $H^{\prime}$ in layer 2.5 on day 35 . The mean thickness of this layer is $\bar{H}=15 \mathrm{dbar}$. Solid contours refer to positive, broken contours to negative anomalies. The zero line is the first solid one. Contour interval is $1 \mathrm{dbar}$. Broken lines indicate positions of cross sections. Cross sections of the layer thickness at (b) $y=13 \mathrm{~km}$ and (c) $y=55 \mathrm{~km}$ on day 35 (solid lines) and day 0 (broken lines). The positions of the sections are indicated in Figs. 3a, 8, 9a, and 10a.

of this layer was 15 dbars. Comparison with Fig. 8 reveals that these positive anomalies are correlated with a local maximum of cyclonic relative vorticity. Hence, they are probably caused by vortex stretching due to local changes of relative vorticity. There are also positive anomalies at $x=160$ and $x=110 \mathrm{~km}$, and negative anomalies at $x=95,125,135$, and $150 \mathrm{~km}$, but here we cannot find any extreme values in the relative vorticity pattern; there must be some other physical mechanism responsible for creating these anomalies. In general, the cross-front layer-thickness modulation exhibits a banded structure with spatial scales on the order of $10 \mathrm{~km}$, from which only the absolute extreme values can be related to extreme values of relative vorticity. The same is valid for the anticyclone section (Fig. 9c). The minimum layer thickness in layers 2.5 and 3.5 at $x=115 \mathrm{~km}$ is correlated with the absolute minimum of relative vorticity at this location, but for 
the high-wavenumber fluctuation of layer thickness no well-matched signal in the cross-front relative vorticity pattern can be found.

\section{8) POTENTIAL VORTICITY}

Initially, the contours of potential vorticity were parallel to the geostrophic velocity vectors, that is, parallel to the streamlines. Figure 10a shows contours of potential vorticity in layer 2.5 on day 35 . The most striking feature is that the potential vorticity contours are wound up counterclockwise in the cyclones and clockwise in the anticyclones, giving the whole pattern a mushroomlike structure. In contrast to the initial situation, the potential vorticity contours are now phase shifted to the left with respect to the streamline pattern. There is no problem comparing the layer 2.5 potential vorticity structure with the streamfunction in the layer above, because the layer 1.5 streamfunction is phase shifted with respect to the streamfunction in layer 2.5 by less than $2 \mathrm{~km}$.

Sections of the cross-front potential vorticity at $y$ $=13$ and $y=55 \mathrm{~km}$ are presented in Figs. 10b,c. In the upper three layers a multiple-banded structure with locally enhanced gradients has developed during the integration of the model. In layer 2.5, for example, there was initially only one potential vorticity maximum at about $x=122 \mathrm{~km}$ and one minimum at $x$ $=135 \mathrm{~km}$. But after 35 days we see in Fig. 10b maximum values at $x=100,125,136$, and $146 \mathrm{~km}$ and minima between them. The gradients on the left-hand side of the left-hand maximum and on the right-hand side of the right-hand minimum are now stronger than the initial cross-front gradients. Qualitatively, the same thing has happened in layers 1.5 and 3.5 and in layers 2.5 and 3.5 in Fig. 10c. Comparison of the layer 2.5 structure with Fig. $10 \mathrm{~b}$ reveals that the minima and maxima are caused by the winding up of potential contours; that is, this mixing process creates a multiple structure of potential vorticity bands. If we compare layers in Figs. 10b,c with corresponding layers in Figs. $9 b, c$ and Fig. 7a,b, we notice that many features of the cross-front layer-thickness modulation and also the positions of the isopycnals' bumps are correlated with the potential vorticity structure.

\section{Local dynamics}

In section $3 \mathrm{~b}$ dynamical structures of the unstable front have been presented. In this section the intention is to interpret these structures by means of dynamical arguments. This can be best done from the viewpoint of conservation of isopycnic potential vorticity

$$
Q=(\zeta+f) / H
$$

where $H=\partial p / \partial s$ represents the layer thickness. Because of $d Q=0$ and $H=H(\zeta, Q)$ individual changes of the layer thickness and the relative vorticity are related by

$$
d H=d \zeta / Q
$$

The first step to gain insight into the model dynamics is to understand which processes lead to the observed pattern of relative vorticity displayed in Fig. 8. A suitable way to do this is to express the relative vorticity in polar coordinates (cf. Olson 1980):

$$
\zeta=\partial v_{a} / \partial R+v_{a} / R
$$

Here $v_{a}$ is the azimuthal velocity and $R$ the distance from the origin. Equation (5) shows that the relative vorticity can be separated into a shear term $\partial v_{a} / \partial R$ and a second term related to the curvature of the flow field. In a purely zonal jet $R$ is infinite; thus, the $\zeta$ contours are parallel to the jet's streamlines. This is the case at the beginning of the model run. But as soon as the jet begins to develop meanders, the curvature vorticity becomes significant and gives a positive contribution to the relative vorticity in the troughs (increase of cyclonic relative vorticity) and a negative contribution in the ridges (increase of anticyclonic relative vorticity). Consequently high relative vorticity values of either sign can be found in locations where the shear vorticity and the curvature vorticity have the same sign, that is, in the troughs on the cyclonically sheared side of the jet and in the ridges on the anticyclonically sheared side. In the situation displayed in Fig. 8, the curvature vorticity and the shear vorticity are on the same order of magnitude because the halfwidth of the jet is comparable to the curvature radius of the flow around the troughs (ridges); thus, $\partial v_{a} / \partial R$ $\approx v_{a} / R$. This explains the nearly vanishing cyclonic relative vorticity north of the ridges and the rather modest anticyclonic relative vorticity south of the troughs (Fig. 11).

An important circumstance generated by the curvature vorticity contribution to the relative vorticity pattern is the fact that in the meandering current the relative vorticity contours now cut across the streamlines (Fig. 11). Hence, there is a nonzero relative vorticity gradient evaluated along any streamline driving the vertical motion (Fig. 6). If we assume to first-order approximation that water parcels are advected along streamlines, then these parcels will "feel" the changing relative vorticity along their path. According to (4) this relative vorticity change will result in a compensating vortex stretching or compression of vortex tubes depending on the sign of the alongstreamline relative vorticity gradient. This gradient is positive in the halfwave between the ridges and the troughs looking downstream and negative elsewhere. Thus, vortex stretching (downwelling) occurs in front of the ridges, and compression of vortex tubes (upwelling) in front of the troughs. Extreme values of vertical motion occur 

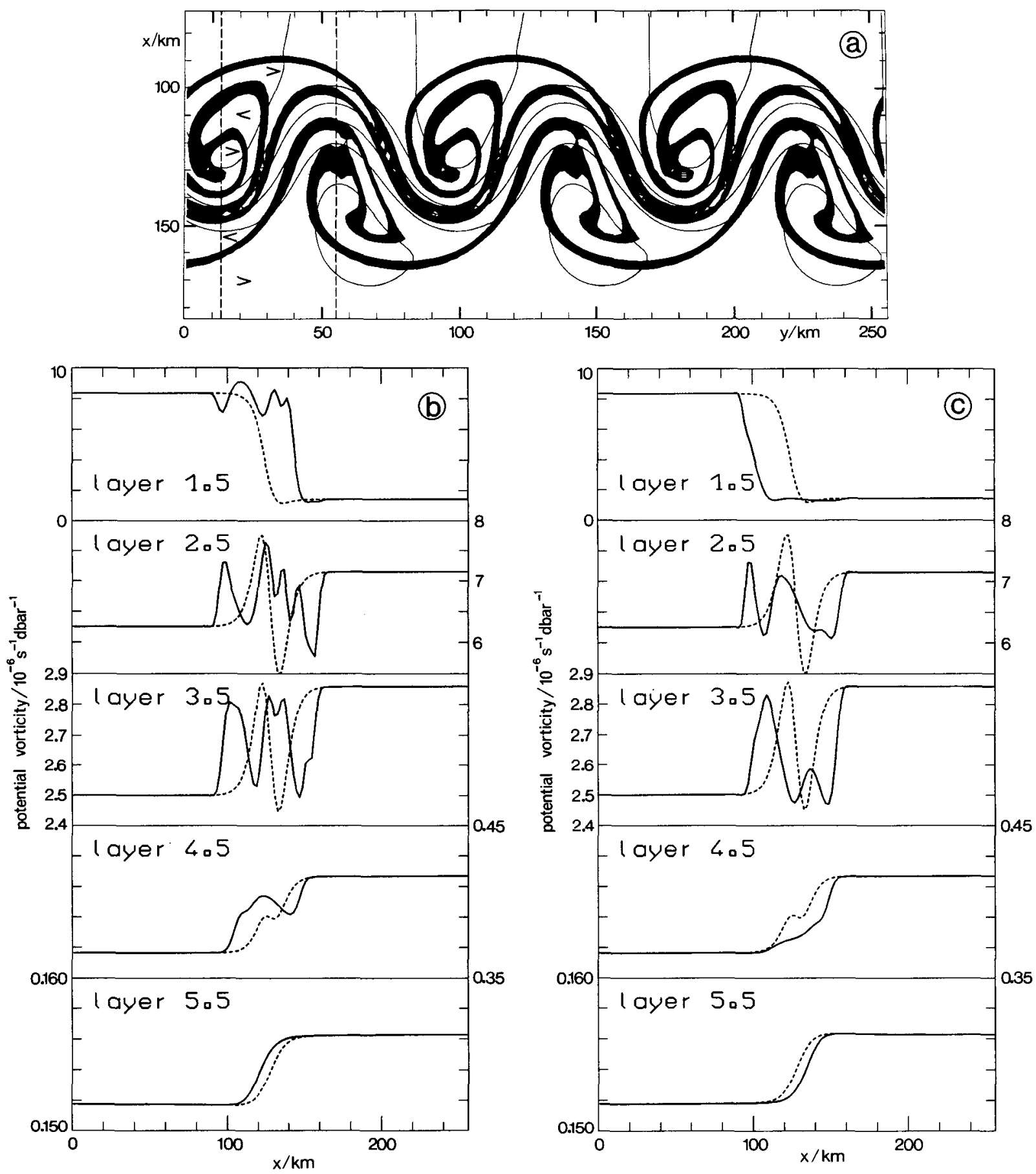

FIG. 10. (a) Potential vorticity in layer 2.5 on day 35 . Only the potential vorticity range between 6.5 and $7 \times 10^{-6} \mathrm{~s}^{-1} \mathrm{dbar}-1$ is shown and colored black. Areas with values $<6.5 \times 10^{-6}$ are marked by $<$; regions with values $>7 \times 10^{-6}$ are marked by $>$. Thin lines are streamlines as shown in Fig. 3a. Broken lines indicate the positions of the cross sections. (b), (c) Cross section of potential vorticity (in $10^{-6} \mathrm{~s}^{-1} \mathrm{dbar}^{-1}$ ) on day 35 (solid lines) and day 0 (broken lines) at (b) at $y=13 \mathrm{~km}$, and (c) at $y=55 \mathrm{~km}$. The positions of the sections are indicated in Figs. 3a, 8, 9a, and 10a.

where $d \zeta / d t$ is strongest, that is, halfway between the troughs and the ridges, where the curvature vorticity changes sign.
A scale analysis confirms the right order of magnitude of the vertical motion diagnosed by the model. The vertical motion at level 2 depends only on the 


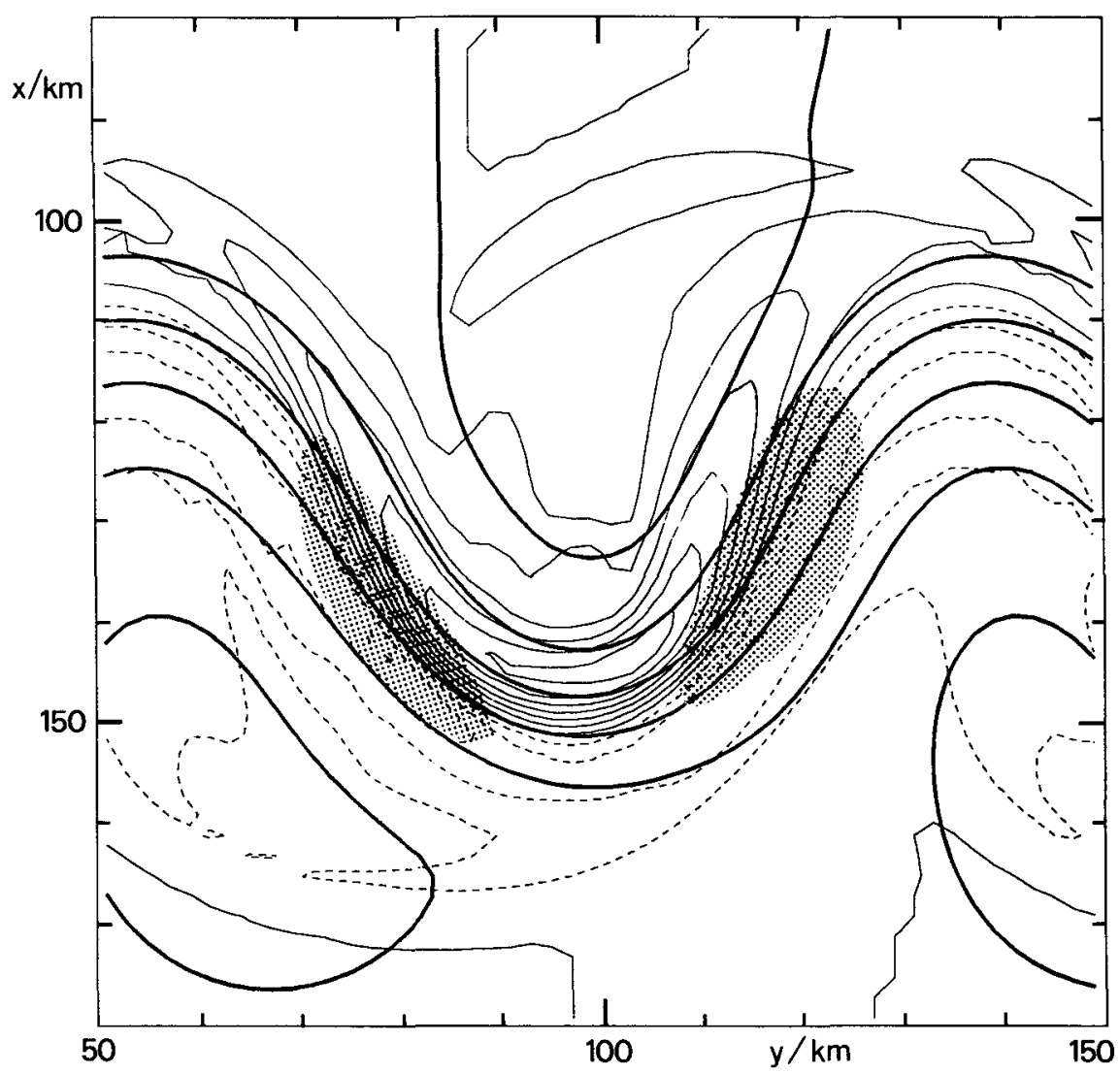

Fig. 11. Overlay of Figs. 3a, 6, and 8. Thick solid lines refer to the streamfunction, thin solid and broken lines to $\zeta / f$. Up- and downwelling areas with $|w| \geqslant 5 \mathrm{~m} \mathrm{~d}^{-1}$ are indicated by shading. Only the range $80 \leqslant x \leqslant 180 \mathrm{~km}$ and $50 \leqslant y \leqslant 150 \mathrm{~km}$ is shown.

thickness variation of layer 1.5 ; thus, $w=d H / d t$ or $w$ $=(d \zeta / d t) / Q$ according to (4), where $H$ is the thickness of layer 1.5. For a jet speed of $\sim 0.4 \mathrm{~m} \mathrm{~s}^{-1}$ a typical time scale for a water particle in the core of the jet to flow along a half-meander wavelength is $T \sim 1$ day, the relative vorticity change along its path is of order $f$, and the potential vorticity is of order $f / H$. Thus, the vertical motion at level 2 is of order $H / T$ or $10 \mathrm{~m} \mathrm{~d}^{-1}$, assuming a typical layer thickness on the order of $10 \mathrm{~m}$.

A direct consequence of the vortex stretching/ compression is the layer-thickness modulation shown in Fig. 9a. In many locations $H^{\prime}$ is quite well correlated to the $\zeta / f$ contours in Fig. 8. This is a circumstance that can also be explained by (4). Water parcels advected along streamlines compensate for individual changes of relative vorticity by changes of the layer thickness. But in certain locations the $H^{\prime}$ contours deviate from the $\zeta / f$ pattern, especially in the interiors of the cyclone and the anticyclone. One reason for this fact might be that Fig. 8 shows $\zeta / f$ in layer 1.5 , whereas the thickness modulation displayed in Fig. 9a occurs in layer 2.5. It has been verified, however, that these deviations also exist within the same layer. Thus, the first-order concept that water parcels are advected along streamlines fails. In contrast to the Lagrangian point of view expressed by (4), local changes of layer thickness can be derived from $d Q / d t=0$ as

$$
\partial H / \partial t=(\partial \zeta / \partial t+H \mathbf{v} \cdot \nabla Q) / Q .
$$

Here, $v$ is the horizontal velocity vector. Equation (6) reveals that in addition to local changes of the relative vorticity [already included in (4)] the advection of potential vorticity has to be taken into account when considering local changes of the layer thickness. A nonzero potential vorticity advection can be due only to ageostrophic advection, because initially the potential vorticity gradient was perpendicular to the geostrophic flow and no potential vorticity gradient existed along the streamlines.

The ageostrophic flow field in layer 1.5 has been evaluated by subtracting the geostrophic flow from the total flow in this layer. The geostrophic flow has been calculated by differentiating the streamfunction displayed in Fig. 3a. The ageostrophic flow, representing the divergent (nonrotational) part of the motion, is 
shown in Fig. 12. Typical velocities are on the order of $10 \mathrm{~mm} \mathrm{~s}^{-1}$. Extreme values of up to $16 \mathrm{~mm} \mathrm{~s}^{-1}$ occur in the troughs and the ridges. In the ridges the flow is positive, that is, to the east. Maximum speeds can be found in proximity of the jet. The same is valid for the troughs, but here the flow has the opposite sign. Comparison of the ageostrophic and the geostrophic flow reveals that in regions of cyclonic streamline curvature the ageostrophic flow is directed opposite to the geostrophic flow (the total flow is subgeostrophic), and where the streamline curvature is anticyclonic, the ageostrophic flow points into the same direction (supergeostrophic). Minimum values are encountered where the sign of the streamline curvature changesand, in fact, far away from the jet. In order to exclude that the ageostrophic flow is an artifact of the initial perturbation, I repeated the model run but used a geostrophically balanced perturbation. The result was that the strength and direction of the divergent flow pattern did not change. In addition, the structure shown in Fig. 12 is well organized in contrast to the randomly distributed initial perturbation.

The divergent flow pattern now helps us to confirm and understand several features described in section $3 b$. The locations of the sinks and sources of the divergent flow field coincide with the position of the vertical motion extrema displayed in Fig. 6. Convergence is associated with downwelling, divergence with upwelling. The deviations of the $H^{\prime}$ pattern from the $\zeta /$ $f$ pattern obviously are due to the cross-jet advection of potential vorticity. This is confirmed by Fig. 10a. Although the initial potential vorticity contours were aligned parallel to the geostrophic flow, they now are tilted and phase shifted with respect to the streamlines. This is a consequence of the time-integrated ageostrophic potential vorticity advection. We can conjecture that the winding up of potential vorticity contours in the cyclones and anticyclones and the enhancement of potential vorticity gradients is also driven by this process. Now we are able to explain the generation of the bumps of the isopycnals in the density sections (Fig. 7). These are layer-thickness anomalies caused by isopycnic advection of potential vorticity. Comparison of Figs. 7, 9b,c, and 10 confirms that the location and the horizontal scale of the bumps in the cross sections coincide with the position and the width of the spiraling bands of potential vorticity. In order to exclude that the bumps are caused by any type of numerical instability or truncation error, I also repeated the model run with half the horizontal resolution; there was no significant difference in comparison with the high-resolution run.

\section{Discussion}

The model results just presented give some insight into mesoscale structures of the seasonal thermocline and their generation due to unstable density fronts. Attention has been focused on dynamical structures associated with a monochromatic unstable wave. Although in reality, oceanic motions extend over the whole wavenumber spectrum, it was not the intention to present a realistic simulation of frontal instability but to relate special patterns created by the model directly to the dynamical background within the unstable wave and to explain their generation by applying simple dynamical concepts. In the following, these results will be compared in particular to results of earlier oceanic and atmospheric models and observations.

The first finding was the different strength of the flow and the width of the jet around cyclones and anticyclones. This is a typical feature of meandering flows, which has been investigated theoretically by Chew (1974). For a frictionless nonlinear current, he derived

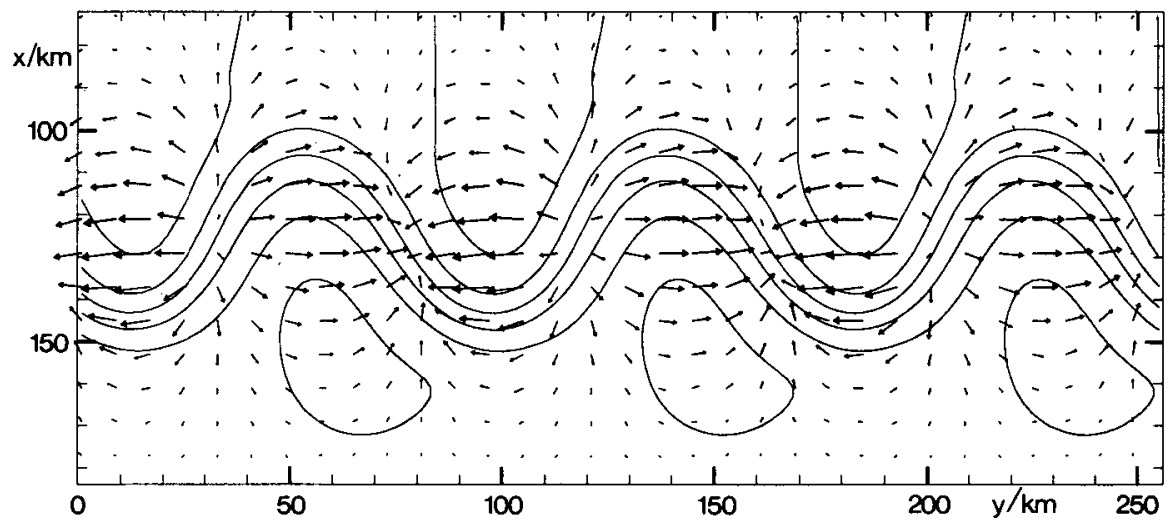

FIG. 12. The divergent part of the flow field in layer 1.5 on day 35 . The length of the arrows and the size of the arrow heads are proportional to the speed. The maximum arrow length refers to a speed of $16 \mathrm{~mm} \mathrm{~s}^{-1}$. Vectors are plotted at every fourth grid point. The solid lines represent the streamfunction displayed in Fig. 3a. 
from the vorticity equation the "turning equation" governing the curvature vorticity change in a meandering current. This change is balanced by four terms, one of which is called the banking term. This term spreads the current out and slows it down when approaching an anticyclonic turn. On the other hand, it accelerates the current and contracts it laterally when approaching a cyclonic turn. In the same paper, Chew proved his theory by deploying parachute drogues in different meandering flows in the Gulf of Mexico. The different strength of the flow has also been measured directly by Osgood et al. (1987) by using a moored current meter array in the South Atlantic Bight.

The model predicts a downwelling up to $10 \mathrm{~m} \mathrm{~d}^{-1}$ behind and an upwelling at the same rate in front of the troughs. This pattern is well known from atmospheric waves (cf. Holton 1979; Gill 1982) with rising air in front of and sinking motion behind the troughs. In the preceding section the vertical motion was explained to be the result of vortex stretching or compression due to individual changes of relative vorticity, which themselves are caused by a relative vorticity gradient along streamlines. In quasigeostrophic theory this mechanism is expressed by the equation

$$
D=\mathbf{v}_{g} \cdot \nabla \zeta /(\zeta+f)
$$

relating the divergence $D$ of the flow field and the geostrophic advection $\mathbf{v}_{g}$ of geostrophic relative vorticity $\zeta_{g}$. From the model results $D$ at level 2 can then be estimated as $3 \times 10^{-6} \mathrm{~s}^{-1}$ assuming a geostrophic jet velocity of $0.4 \mathrm{~m} \mathrm{~s}^{-1}$, a relative vorticity change of $f$ along a path of $60 \mathrm{~km}$ (i.e., between crest and trough). The vertical velocity scale $W$ can be estimated as $W$ $=D \cdot H$, where $H$ is the layer thickness. For a mean thickness $H$ of about $40 \mathrm{~m}$ (layer 1.5 ) $W$ yields as 11.5 $\mathrm{m} \mathrm{d}^{-1}$, which is close to the maximum model vertical velocity. The foregoing method has also been used by Leach (1987) to diagnose the vertical velocity field from direct horizontal current measurements at the North Atlantic polar front. Typical vertical velocities in his study were $6 \mathrm{~m} \mathrm{~d}^{-1}$ using $H=50 \mathrm{~m}$. Direct measurements of vertical velocities in Gulf Stream meanders have been carried out by Chew et al. (1985) using drogues deployed at a depth of about $40 \mathrm{~m}$. They measured vertical velocities of about $10 \mathrm{~m} \mathrm{~d}^{-1}$ with upwelling in front of troughs and downwelling behind troughs. Levine et al. (1986) deployed isopycnal-following floats at a depth of about $400 \mathrm{~m}$ in the Gulf Stream. From simultaneous hydrostations and XBT drops along the float trajectory they could show that a Lagrangian water volume converges and stretches vertically when approaching a cyclonic turn, and when approaching an anticyclonic turn, the column diverges and contracts. During periods of vortex stretching the float descended (downwelling), while during episodes of contraction the float was shoaling. The position of the vertical velocity extrema was also consistent with findings of Osgood et al. (1987), Garvine (1988), Bower (1989), and Bower and Rossby (1989).

In the model $I$ have identified mechanisms inducing cross-front modulations of layer thickness: in an Eulerian sense, local changes of layer thickness can be due to vortex stretching caused by local changes of relative vorticity or advection of different potential vorticity [cf. (6)]. From a Lagrangian point of view, only vortex stretching/compression caused by relative vorticity changes along the path of a water particle can lead to thickness modulations [cf. (4)]. The major part of the observational literature is Eulerian. Hence, in order to decide which term has lead to the observed modulations of layer thickness (or vertical stability as an equivalent quantity), it is necessary to gain information on the relative and potential vorticity distribution and to relate these to the structure of a front and the frontal jet. But this requires high-resolution synoptic measurements of the density and velocity field including repeat surveys, which is a complicated and expensive task to do in the ocean. Only some elements of this complex work have been done, and we are left to relate these fragments to the model results. Changes of vertical stability across an oceanic front in the Mediterranean Sea with higher stability on the anticyclonically sheared side of the jet have been observed by Johannessen et al. (1977). Fischer et al. (1989) mapped the distribution of isopycnal potential vorticity in the seasonal thermocline at the North Atlantic polar front and found significant changes of layer thickness across the front, but in contrast to my model results (cf. Fig. $9 a$ ), they encountered positive anomalies in the ridge and negative anomalies in the trough of a meander. This is not a contradiction because ( 7 ) does not give any information on the thickness itself, but only on the temporal change. In principle, the observed pattern could also have been produced by the model if the prescribed initial potential vorticity gradient in layer 2.5 had a different sign. Fischer et al. also evaluated various terms of potential vorticity advection, but they were not able to compare these terms with local changes of relative vorticity because they had in general no information on temporal evolutions. This example shows that from a one-time survey it is impossible to isolate the physical mechanism that might have caused the thickness anomaly. More information can be drawn from Lagrangian floats launched in a meandering current. Bower (1989) and Schultz and Rossby (1988) found stability changes along a float trajectory in the Gulf Stream clearly related to changing lateral shear and curvature vorticity.

This model has demonstrated that there is a crossfront ageostrophic transport of potential vorticity. Potential vorticity contours "leave" the jet on the cyclonically and anticyclonically sheared side of the jet when approaching an anticyclonic turn (Fig. 10a), which must be due to the divergent flow there. This 
cross-front exchange of matter has also been proved experimentally by Bower and Rossby (1989). They launched a set of RAFOS floats in the Gulf Stream, many of which escaped from the stream after a mean residence time of three weeks. From these trajectories they constructed a conceptual model in which water parcels escape to the north as they approach an anticyclonic turn and to the south when approaching a cyclonic turn. Unfortunately, only the first observation matches these model results. This difference could be caused by the limited amount of observations or by the fact that the authors identified the jet's path by the sea surface temperature pattern, which may be phase shifted with respect to the current.

In this model the potential vorticity is wound up in cyclones and anticyclones after having escaped from the jet. This feature is a familiar phenomenon shown in many oceanic models (cf. Ikeda and Emery 1984; Mied et al. 1991). In the observational literature, information about the potential vorticity structure is nearly always missing, but from satellite images the winding up of isotherms (Viehoff and Fischer 1988; Mied et al. 1991) and analogous structures in sea surface color (Gower et al. 1980) are well known. Though a priori these quantities are not correlated with potential vorticity, a qualitative comparison of the spiral pattern of these quantities is at least partially justified by the fact that sea surface color and temperature are in some ways characteristic of a water mass like potential vorticity. I have shown that the spiral-shaped advection of potential vorticity streamers causes thickness anomalies that may appear as bumps in the depth of isopycnals. It is difficult to observe these bumps in the ocean because of the contamination of the density field by internal waves. But there is some observational evidence for the existence of these bumps and their relation to potential vorticity streamers, for example, in oceanic eddies (cf. Olson 1980). In his Fig. 1, bumps with horizontal scales of about $20 \mathrm{~km}$ can be seen on density surfaces $\sigma_{\theta}=26.4$ and $27.8 \mathrm{~kg} \mathrm{~m}^{-3}$ at about $50-60-\mathrm{km}$ radial distance from the ring center. The bumps on the upper isopycnals seem to be related to thickness anomalies in the layer bounded by the isopycnal surfaces 26.4 and $26.8 \mathrm{~kg} \mathrm{~m}^{-3}$. His Fig. 7a indeed reveals a streamer with different potential vorticity just at the same radial distance.

\section{Summary and conclusions}

Results of a three-dimensional primitive equation model simulating structures produced by mesoscale turbulence in the seasonal thermocline have been presented. In the model, mesoscale turbulent motions are generated by means of an unstable potential vorticity front. This front exhibits features, many of which are qualitatively known from other models and observa- tions. In order to present a quantitative description, dynamical structures of the most unstable wave growing on this front have been investigated, which in this study has a wavelength of $85.3 \mathrm{~km}$. Conservation of isopycnic potential vorticity is guaranteed by using hybrid coordinates, remaining isopycnic everywhere during model integration. This, in connection with the lower-resolution run, which gave the same answers, implies that the locations and the magnitude of quantities evaluated in the model are reliable.

The model relates the vertical motion to the streamline curvature. Vertical motion driven by the relative vorticity gradient along any trajectory is of order $1 \mathrm{~m}$ $\mathrm{d}^{-1}$, extreme values are more than $10 \mathrm{~m} \mathrm{~d}^{-1}$ in places where the streamline curvature changes sign and the frontal jet is strongest. This may help biologists locate regions where nutrients are resupplied into the euphotic zone. As the gradient increases during amplification of the instability due to the growing curvature contribution, values even greater than $10 \mathrm{~m} \mathrm{~d}^{-1}$ seem to be possible. The horizontal extent of the upwelling region is about half the meander wavelength, in the present case around $40 \mathrm{~km}$. For a phytoplankton cell advected by the frontal jet this means a residence time of a few days in the upwelling region, if we assume the advection to be on the order of $0.1 \mathrm{~m} \mathrm{~s}^{-1}$ or $10 \mathrm{~km} \mathrm{~d}^{-1}$-enough time for a few additional cell divisions due to better light conditions (Raymont 1980).

The model reveals zones of different static stability across the frontal jet. This makes shear instability and turbulent vertical mixing (entrainment) more likely in regions of cyclonic relative vorticity and is important for the passage of internal waves through frontal zones. The model describes the strength and direction of the ageostrophic flow. This helps to interpret float trajectories and to estimate cross-frontal exchange rates of mass and heat. The model simulates the winding up of potential vorticity and any other correlated conservative quantity and explains the generation of multiple fronts and density finestructure in terms of potential vorticity advection. This process helps the potential enstrophy cascade to penetrate further into the mesoscale waveband toward higher wavenumbers, a process that could not be achieved by two-dimensional frontogenesis models.

Acknowledgments. Several elements of this paper can be traced back to my Ph.D. thesis. I would like to express my gratitude to my thesis advisor, Prof. J. D. Woods, who recognized the importance of mesoscale turbulence for the structure of the upper ocean. His basic ideas and early conceptual models of mesoscale frontal dynamics formed the scientific background for my thesis. I also would like to thank K. Speer and the anonymous reviewers for helpful comments on an early version of the paper and $\mathrm{C}$. Brückner for preparing the figures. 


\section{REFERENCES}

Bleck, R., and D. B. Boudra, 1981: Initial testing of a numerical ocean circulation model using a hybrid (quasi-isopycnic) vertical coordinate. J. Phys. Oceanogr., 11, 755-770.

- , and - -1986 : Wind-driven spin-up in eddy-resolving models formulated in isopyenic and isobaric coordinates. $J$. Geophys. Res., 91(C6), 7611-7621.

$\longrightarrow$, R. Onken, and J. D. Woods, 1988: A two-dimensional model of mesoscale frontogenesis in the ocean. Quart. J. Roy. Meteor. Soc. 114, 347-371.

Boudra, D. B., R. Bleck, and F. Schott, 1988: A numerical model of instabilities in the Florida Current. J. Mar. Res., 46, 715-751.

Bower, A. S., 1989: Potential vorticity balances and horizontal divergences along particle trajectories in Gulf Stream meanders east of Cape Hatteras. J. Phys. Oceanogr., 19, 1669-1681.

- , and T. Rossby, 1989: Evidence of cross-frontal exchange processes in the Gulf Stream based on isopycnal RAFOS float data. J. Phys. Oceanogr., 19, 1177-1190.

Charney, J. G., 1971: Geostrophic turbulence. J. Atmos. Sci., 28, $1087-1095$.

Chew, F., 1974: The turning process in meandering currents: A case study. J. Phys. Oceanogr., 14, 27-57.

- J. M. Bane, and D. A. Brooks, 1985: On vertical motion, divergence and the thermal wind balance in cold-dome meanders: A diagnostic study. J. Geophys. Res., 90(C2), 3173-3183.

Cox, M. D., 1987: An eddy-resolving model of the ventilated thermocline. J. Phys. Oceanogr., 17, 1044-1056.

Fischer, J., H. Leach, and J. D. Woods, 1989: A synoptic map of isopycnic potential vorticity in the seasonal thermocline. J. Phys. Oceanogr., 19, 519-531.

Flament, P., L. Armi, and L. Washburn, 1985: The evolving structure of an upwelling filament. J. Geophys. Res., 90(C6), 11 76511778.

Garvine, R. W., 1988: Flow field properties of long, propagating frontal waves. $J$. Phys. Oceanogr., 18, 788-792.

Gill, A. E., 1982: Atmosphere-Ocean Dynamics. Academic Press, $662 \mathrm{pp}$.

Gower, J. F. R., K. L. Denman, and R. J. Holyer, 1980: Plankton patchiness indicates the fluctuation spectrum of mesoscale oceanic structure. Nature, 288, 157-159.

Holton, J., 1979: Dynamic Meteorology. 2d ed., Academic Press, 393 pp.

Ikeda, M., 1981: Meanders and detached eddies of a strong eastwardflowing jet using a two-layer quasigeostrophic model. J. Phys. Oceanogr., 11, 526-540.

of meanders in the California Current system off Oregon and Northern California. J. Phys. Oceanogr., 14, 1434-1450.

Johannessen, O. M., D. Good, and G. Smallenburger, 1977: Observations of an oceanic front in the Ionian Sea during early winter 1970. J. Geophys. Res., 82, 1381-1391.

Joyce, T. M., 1977: A note on the lateral mixing of water masses. $J$ Phys. Oceanogr., 7, 626-629.
Kielmann, J., and R. H. Käse, 1987: Numerical modeling of meander and eddy formation in the Azores Current frontal zone. J. Phys. Oceanogr., 17, 529-541.

Killworth, P. D., 1980: Barotropic and baroclinic instability in rotating stratified fluids. Dyn. Atmos. Oceans., 4, 143-184.

, N. Paldor, and M. Stern, 1984: Wave propagation and growth on a surface front in a two-layer geostrophic current. J. Mar. Res., 42, 761 785 .

Leach, H., 1987: The diagnosis of synoptic-scale vertical motion in the seasonal thermocline. Deep-Sea Res., 34, 2005-2017.

Levine, E. R., D. N. Connors, P. C. Cornillon, and H. T. Rossby, 1986: Gulf Stream kinematics along an isopycnal float trajectory. J. Phys. Oceanogr., 16, 1317-1328.

McWilliams, J. C., 1985: Submesoscale coherent vortices in the ocean. Rev. Geophys., 23, 165-182.

Mied, R. P., J. C. McWilliams, and G. J. Lindemann, 1991: The generation and evolution of mushroom-like vortices. J. Phys. Oceanogr., 21, 489-510.

Oey, L.-Y., 1988: A model of Gulf Stream frontal instabilities, meanders and eddies along the continental slope. J. Phys. Oceanogr., $18,211-229$.

Olson, D. B., 1980: The physical oceanography of two rings observed by the Cyclonic Ring Experiment. Part II: Dynamics. J. Phys. Oceanogr., 10, 514-528.

Osgood, K. E., J. M. Bane, Jr,, and W. K. Dewar, 1987: Vertical velocities and dynamical balances in Gulf Stream meanders. $J$. Geophys. Res., 92(C12), 13 029-13 040.

Pedlosky, J., 1979: Geophysical Fluid Dynamics. Springer-Verlag, $624 \mathrm{pp}$.

Raymont, J. E. G., 1980: Plankton and Productivity in the Oceans. Vol. 1: Phytoplankton, 2d ed., Pergamon, $489 \mathrm{pp}$.

Rhines, P. B., 1979: Geostrophic turbulence. Ann. Rev. Fluid Mech., 11, 401-441.

Salmon, R., 1980: Baroclinic instability and geostrophic turbulence. Geophys. Astrophys. Fluid Dyn., 15(3/4), 167-211.

Saltzman, B., and C.-M. Tang, 1975: Formation of meanders, fronts, and cut-off thermal pools in a baroclinic ocean current. J. Phys. Oceanogr., 5, 86-92.

Schultz, K. L., and T. Rossby, 1988: Cross- and downstream variability of static stability in the Gulf Stream. $J$. Phys. Oceanogr., 18, 1933-1937.

Viehoff, T., and J. Fischer, 1988: Satellite sea surface temperature at the North Atlantic Polar front related to high-resolution towed conductivity-temperature-depth data. J. Geophys. Res., 93( $\mathrm{C} 12), 15551-15560$.

Wood, R. A., 1988: Unstable waves on oceanic fronts: Large amplitude behavior and mean flow generation. J. Phys. Oceanogr. 18, 775-787.

Woods, J. D., 1980: Do waves limit turbulent diffusion in the ocean? Nature, 288, 219-224.

_ R. Onken, and J. Fischer, 1986: Thermohaline intrusions created isopycnically at oceanic fronts are inclined to isopycnals. Nature, 322, 446-449. 